\title{
New Formulas for the Mayer and Ree-Hoover Weights of Infinite Families of Graphs
}

\author{
Amel Kaouche \\ Université de Moncton, Campus d'Edmundston, d'Edmundston, Canada \\ Email: amel.kaouche@umoncton.ca
}

How to cite this paper: Kaouche, A. (2019) New Formulas for the Mayer and Ree-Hoover Weights of Infinite Families of Graphs. World Journal of Engineering and Technology, 7, 283-292. https://doi.org/10.4236/wjet.2019.72019

Received: March 6, 2019

Accepted: April 8, 2019

Published: April 15, 2019

\begin{abstract}
The virial expansion, in statistical mechanics, makes use of the sums of the Mayer weight of all 2-connected graphs on $\mathrm{n}$ vertices. We study the Second Mayer weight $\omega_{M}(\mathrm{c})$ and the Ree-Hoover weight $\omega R H(\mathrm{c})$ of a 2-connected graph $c$ which arise from the hard-core continuum gas in one dimension. These weights are computed using signed volumes of convex polytopes naturally associated with the graph c. In the present work, we use the method of graph homomorphisms, to give new formulas of Mayer and Ree-Hoover weights for special infinite families of 2-connected graphs.
\end{abstract}

\section{Keywords}

Mayer Weight, Ree-Hoover Weight, Graph Invariants, Virial Expansion

\section{Introduction}

Graph weights can be defined as functions on graphs taking scalar or polynomial values and which are invariant under isomorphism. In the context of a non-ideal gas in a vessel $V \subseteq \mathbb{R}^{d}$, the Second Mayer weight $w_{M}(c)$ of a connected graph $\mathcal{C}$, over the set $[n]=\{1,2, \ldots, n\}$ of vertices, is defined by (see [1] [2] [3] [4])

$$
w_{M}(c)=\int_{\left(\mathbb{R}^{d}\right)^{n-1}} \prod_{\{i, j\} \in c} f\left(\left\|\overrightarrow{x_{i}}-\overrightarrow{x_{j}}\right\|\right) d \overrightarrow{x_{1}} \cdots d \overrightarrow{x_{n-1}}, \quad \overrightarrow{x_{n}}=0,
$$

where $\overrightarrow{x_{1}}, \ldots, \overrightarrow{x_{n}}$ are variables in $\mathbb{R}^{d}$ representing the positions of $n$ particles in $V(V \rightarrow \infty)$, the value $\overrightarrow{x_{n}}=0$ being arbitrarily fixed, and where $f=f(r)$ is real-valued function associated with the pairwise interaction potential of the particles, see [4] [5]. The Mayer weight occur in the so-called virial expansion proposed by Kamerlingh Onnes in 1901

$$
\frac{P}{k T}=\rho+\beta_{2} \rho^{2}+\beta_{3} \rho^{3}+\cdots
$$


where $k$ is a constant, $T$ is the temperature and $\rho$ is the density. Indeed, it can be shown that

$$
\beta_{n}=\frac{1-n}{n !}|\mathcal{B}[n]|_{w_{M}},
$$

where $\mathcal{B}[n]$ denote the set of 2 -connected graphs over $[n]$ and $|\mathcal{B}[n]|_{w_{M}}$ is the total sum of weights of 2-connected graphs over $[n]$. In order to compute this expansion numerically, Ree and Hoover [6] introduced a modified weight denoted by $w_{R H}(b)$, for 2 -connected graphs $b$, which greatly simplifies the computations. It is defined by

$$
w_{R H}(b)=\int_{\left(\mathbb{R}^{d}\right)^{n-1}} \prod_{\{i, j\} \in b} f\left(\left\|\overrightarrow{x_{i}}-\overrightarrow{x_{j}}\right\|\right) \prod_{\{i, j\} \notin b} \bar{f}\left(\left\|\overrightarrow{x_{i}}-\overrightarrow{x_{j}}\right\|\right) d \overrightarrow{x_{1}} \cdots d \overrightarrow{x_{n-1}}, \quad \overrightarrow{x_{n}}=0,
$$

where $\bar{f}(r)=1+f(r)$. Using this new weight, Ree and Hoover [6] [7] [8] and later Clisby and McCoy [9] [10] have computed the virial coefficients $\beta_{n}$, for $n$ up to 10 , in dimensions $d \leq 8$, in the case of the hard-core continuum gas, that is when the interaction is given by

$$
f(r)=-\chi(r<1), \bar{f}(r)=\chi(r \geq 1),
$$

where $\chi$ denote de characteristic function. In this paper we study graph weights $w_{M}(b)$ and $w_{R H}(b)$ in the context of the hard core continuum gas, defined by (1.4), in dimension $d=1$. The values $w_{M}(c)$ and $w_{R H}(c)$ for all 2-connected graphs $c$ of size at most 8 are given in [1] [11]. In Section 2.1, we look at the case of the hard-core continuum gas in one dimension in which the Mayer weight turns out to be a signed volume of a convex polytope $\mathcal{P}(c)$ naturally associated with the graph $c$. An alternate useful tool, a decomposition of the polytope $\mathcal{P}(c)$ into a certain number of $(n-1)$-dimensional simplices, of volume 1/(n-1)! is exploited in Section 2.2. This method was introduced in [4] and was adapted in [3] to the context of Ree-Hoover weights and is called the method of graph homomorphisms. The explicit computation of Mayer or Ree-Hoover weights of particular graphs is very difficult in general and have been made for only certain specific families of graphs (see [2] [3] [4] [12] [13] [14]). In the present paper we extend this list to other graphs. We give new explicit formulas of the Mayer and Ree-Hoover weights for special infinite families of graphs in Section 2.3.

\section{Mayer and Ree-Hoover Weights}

\subsection{Hard-Core Continuum Gas in One Dimension}

Consider $n$ hard particles of diameter 1 on a line segment. The hard-core constraint translates into the interaction potential $\varphi$, with $\varphi(r)=\infty$, if $r<1$, and $\varphi(r)=0$, if $r \geq 1$, and the Mayer function $f$ and the Ree-Hoover function $\bar{f}$ are given by (1.4). Hence, we can write the Mayer weight function $w_{M}(c)$ of a connected graph $c$ as

$$
w_{M}(c)=(-1)^{e(c)} \int_{\mathbb{R}^{n-1}} \prod_{\{i, j\} \in c} \chi\left(\left|x_{i}-x_{j}\right|<1\right) d x_{1} \ldots d x_{n-1}, \quad x_{n}=0,
$$

and the Ree-Hoover's weight function $w_{R H}(c)$ of a 2-connected graph $c$ as 


$$
w_{R H}(c)=(-1)^{e(c)} \int_{\mathbb{R}^{n-1}} \prod_{\{i, j\} \in c} \chi\left(\left|x_{i}-x_{j}\right|<1\right) \prod_{\{i, j\} \notin c} \chi\left(\left|x_{i}-x_{j}\right|>1\right) d x_{1} \ldots d x_{n-1},
$$

with $x_{n}=0$ and where $e(c)$ is the number of edges of $c$.

Note that $w_{M}(c)=(-1)^{e(c)} \operatorname{Vol}(\mathcal{P}(c))$, where $\mathcal{P}(c)$ is the polytope defined by

$$
\mathcal{P}(c)=\left\{X \in \mathbb{R}^{n}\left|x_{n}=0,\right| x_{i}-x_{j} \mid<1 \quad \forall\{i, j\} \in c\right\} \subseteq \mathbb{R}^{n-1} \times\{0\} \subseteq \mathbb{R}^{n},
$$

where $X=\left(x_{1}, \ldots, x_{n}\right)$. Similarly, $w_{R H}(c)=(-1)^{e(c)} \operatorname{Vol}\left(\mathcal{P}_{R H}(c)\right)$, where $\mathcal{P}_{R H}(c)$ is the union of polytopes defined by

$$
\mathcal{P}_{R H}(c)=\left\{X \in \mathbb{R}^{n}\left|x_{n}=0,\right| x_{i}-x_{j}|<1 \quad \forall\{i, j\} \in c,| x_{i}-x_{j} \mid>1 \quad \forall\{i, j\} \in \bar{c}\right\} .
$$

\subsection{Graph Homomorphisms}

The method of graph homomorphisms was introduced in [4] for the exact computation of the Mayer weight $w_{M}(b)$ of a 2-connected graph $b$ in the context of hard-core continuum gases in one dimension and was adapted in [3] to the context of Ree-Hoover weights. Since $w_{M}(b)=(-1)^{e(b)} \operatorname{Vol}(\mathcal{P}(b))$, the computation of $w_{M}(b)$ is reduced to the computation of the volume of the polytope $\mathcal{P}(b)$ associated to $b$. In order to evaluate this volume, the polytope $\mathcal{P}(b)$ is decomposed into $v(b)$ simplices which are all of volume $1 /(n-1)$ !. This yields $\operatorname{Vol}(\mathcal{P}(b))=v(b) /(n-1) !$. The simplices are encoded by a diagram associated to the integral parts and the relative positions of the fractional parts of the coordinates $x_{1}, \ldots, x_{n}$ of points $X \in \mathcal{P}(b)$ (see [3] [4] for more details).

Lemma 1. ([3]). Suppose that $g$ is a graph over $[n]$ and $i, j \in[n-1]$ are such that $g$ does not contain the edge $\{n, i\}$ but contains the edges $\{i, j\}$ and $\{n, j\}$. In this case, any RH-configuration $(h, \beta) \quad\left(\right.$ with $\left.h_{n}=-1, \beta(n)=n\right)$ satisfies either one of the following conditions.

1) $h_{i}=1, h_{j}=0$ and $\beta(i)<\beta(j)$,

2) $h_{i}=-2, h_{j}=-1$ and $\beta(i)>\beta(j)$.

\subsection{Mayer and Ree-Hoover Weights of Some Infinite Families of Graphs}

Here are some of our results concerning new explicit formulas for the Ree-Hoover weight of certain infinite families of graphs. These were first conjectured from numerical values using Ehrhart polynomials. Their proofs use the techniques of graph homorphisms. We also give explicit formulas for the Mayer weight of the same infinite families of graphs. In order to do so, we use the following formula (see [3] for more details)

$$
\left|w_{M}(b)\right|=\sum_{b \subseteq d \subseteq K_{n}}\left|w_{R H}(d)\right| .
$$

\subsubsection{The Mayer and Ree-Hoover Weight of the Graph $K_{n} \backslash\left(C_{4} \cdot \cdot S_{2}\right)$}

Let $C_{4} \cdot \cdot S_{2}$ denote the graph obtained by identifying two non adjacent vertices of the graph $C_{4}$ with the extremities of a 2-star graph, where $C_{4}$ is the cycle with 4 vertices and $S_{k}$ denote the $k$-star graph with vertex set $[k+1]$ and edge set $\{\{1,2\},\{1,3\}, \ldots,\{1, k+1\}\}$. See Figure 1 . 


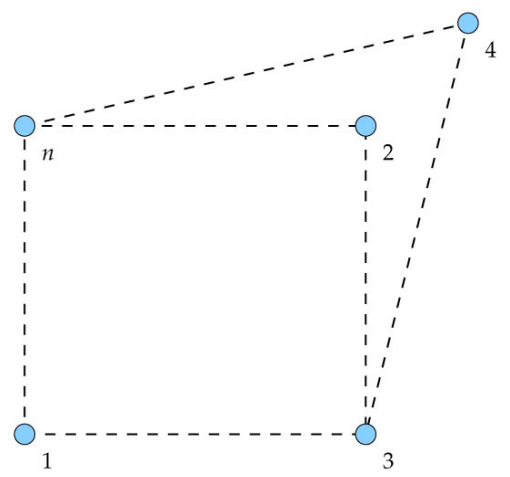

Figure 1. The graph $C_{4} \cdot S_{2}$.

Proposition 1. For $n \geq 7$, we have

$$
\begin{aligned}
\left|w_{R H}\left(K_{n} \backslash\left(C_{4} \cdot \cdot S_{2}\right)\right)\right| & =\frac{24}{(n-1)(n-2)(n-3)(n-4)} . \\
\left|w_{M}\left(K_{n} \backslash\left(C_{4} \cdot \cdot S_{2}\right)\right)\right|= & n+\frac{12}{n-1}+\frac{36}{(n-1)(n-2)} \\
& +\frac{72}{(n-1)(n-2)(n-3)}+\frac{72}{(n-1) \ldots(n-4)} .
\end{aligned}
$$

Proof. We can assume that the missing edges are $\{1, n\},\{2, n\},\{4, n\}$, $\{1,3\},\{3,4\}$ and $\{2,3\}$ (see Figure 1 ).

According to Lemma 1 there are four possibilities for $h$ :

- $h_{1}=h_{2}=h_{4}=1$ and $h_{n}=-1$ and all other $h_{i}=0$, so that $\beta(3)=1$ and $(\beta(1), \beta(2), \beta(4))$ must be a permutation of $\{2,3,4\}$.

- $h_{1}=h_{2}=h_{4}=-2$ and all other $h_{i}=-1$, so that $\beta(3)=n-1$ and $(\beta(1), \beta(2), \beta(4))$ must be a permutation of $\{n-2, n-3, n-4\}$.

- $h_{1}=h_{2}=h_{4}=1$ and $h_{3}=h_{n}=-1$ and all other $h_{i}=0$, so that $\beta(3)=n-1$ and $(\beta(1), \beta(2), \beta(4))$ must be a permutation of $\{1,2,3\}$.

- $h_{3}=0$ and $h_{1}=h_{2}=h_{4}=-2$ and all other $h_{i}=-1$, so that $\beta(3)=1$ and $(\beta(1), \beta(2), \beta(4))$ must be a permutation of $\{n-1, n-2, n-3\}$.

In each case $\beta$ can be extended in $(n-5)$ ! ways, giving the possible relative positions of the $(n-5) \quad x_{i}$ (see Figure 2). So, there are $4 \cdot 3 !(n-5)$ ! $\mathrm{RH}$ - configurations $(h, \beta)$. Which concludes the proof of (2.6).

The over graphs of $K_{n} \backslash\left(C_{4} \cdot \cdot S_{2}\right)$ whose Ree-Hoover weight is not zero and their multiplicities are given by

$$
\begin{aligned}
\left|w_{M}\left(K_{n} \backslash\left(C_{4} \cdot \cdot S_{2}\right)\right)\right|= & \left|w_{R H}\left(K_{n}\right)\right|+6\left|w_{R H}\left(K_{n} \backslash S_{1}\right)\right|+9\left|w_{R H}\left(K_{n} \backslash S_{2}\right)\right| \\
& +2\left|w_{R H}\left(K_{n} \backslash S_{3}\right)\right|+3\left|w_{R H}\left(K_{n} \backslash C_{4}\right)\right| \\
& +12\left|w_{R H}\left(K_{n} \backslash\left(S_{1}-S_{1}\right)\right)\right|+6\left|w_{R H}\left(K_{n} \backslash\left(S_{1}-S_{2}\right)\right)\right| \\
& +6\left|w_{R H}\left(K_{n} \backslash C_{4} \cdot S_{1}\right)\right|+\left|w_{R H}\left(K_{n} \backslash C_{4} \cdot \cdot S_{2}\right)\right| .
\end{aligned}
$$

We conclude using Proposition (1) and Propositions (19)-(23) of [3].

\subsubsection{The Mayer and Ree-Hoover Weight of the Graph $K_{n} \backslash\left(S_{j} \cdot C_{4} \cdot \cdot S_{2}\right)$}

Let $S_{j} \cdot C_{4} \cdot \cdot S_{2}$ denote the graph obtained by identifying one vertex, with 

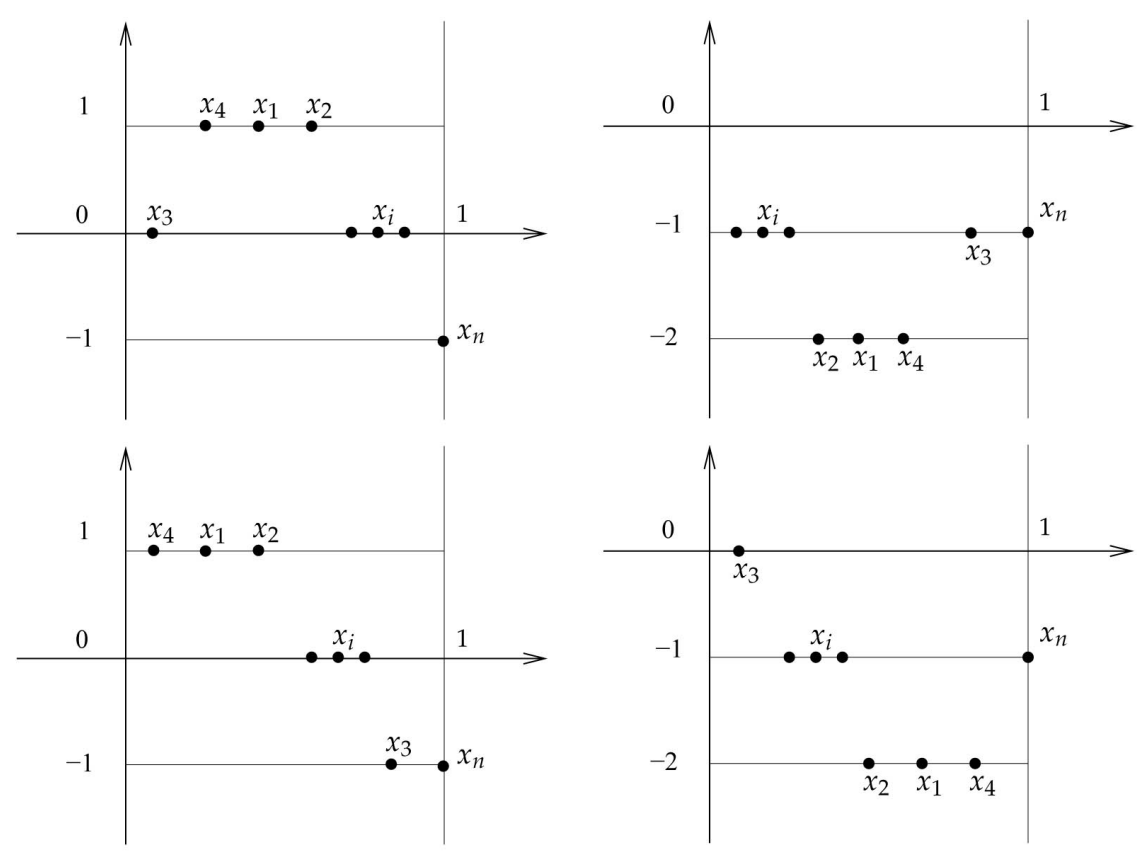

Figure 2. Fractional representation of a simplicial subpolytope of $\mathcal{P}_{R H}\left(K_{n} \backslash\left(C_{4} \cdot \cdot S_{2}\right)\right)$.

degree two, of the graph $\left(C_{4} \cdot \cdot S_{2}\right)$ with a center of a $j$-star. See Figure 3 for an example.

Let us start with the case $S_{1} \cdot C_{4} \cdot \cdot S_{2}$.

Proposition 2. For $n \geq 7$, we have

$$
\begin{aligned}
\left|w_{R H}\left(K_{n} \backslash\left(S_{1} \cdot C_{4} \cdot S_{2}\right)\right)\right| & =\frac{8}{(n-1)(n-2)(n-3)(n-4)(n-5)} . \\
\left|w_{M}\left(K_{n} \backslash\left(S_{1} \cdot C_{4} \cdot \cdot S_{2}\right)\right)\right|= & n+\frac{14}{n-1}+\frac{44}{(n-1)(n-2)}+\frac{92}{(n-1) \ldots(n-3)} \\
& +\frac{104}{(n-1) \ldots(n-4)}+\frac{32}{(n-1) \ldots(n-5)} .
\end{aligned}
$$

Proof. We can assume that the missing edges are $\{1, n\},\{2, n\},\{4, n\}$, $\{1,3\},\{1,5\},\{3,4\}$ and $\{2,3\}$ (see Figure 4).

According to Lemma 1 there are four possibilities for $h$ :

- $h_{1}=h_{2}=h_{4}=1$ and $h_{n}=-1$ and all other $h_{i}=0$, so that $\beta(3)=1$ and $(\beta(2), \beta(4))$ must be a permutation of $\{2,3\}$ and $\beta(5)=4$ and $\beta(1)=5$.

- $h_{1}=h_{2}=h_{4}=-2$ and all other $h_{i}=-1$, so that $\beta(3)=n-1$ and $(\beta(2), \beta(4))$ must be a permutation of $\{n-2, n-3\}$ and $\beta(5)=n-4$ and $\beta(1)=n-5$.

- $h_{1}=h_{2}=h_{4}=1$ and $h_{3}=h_{n}=-1$ and all other $h_{i}=0$, so that $\beta(3)=n-1$ and $(\beta(2), \beta(4))$ must be a permutation of $\{2,3\}$ and $\beta(5)=3$ and $\beta(1)=4$.

- $h_{3}=0$ and $h_{1}=h_{2}=h_{4}=-2$ and all other $h_{i}=-1$, so that $\beta(3)=1$ and $(\beta(2), \beta(4))$ must be a permutation of $\{n-2, n-3\}$ and $\beta(5)=n-3$ and $\beta(1)=n-4$. 


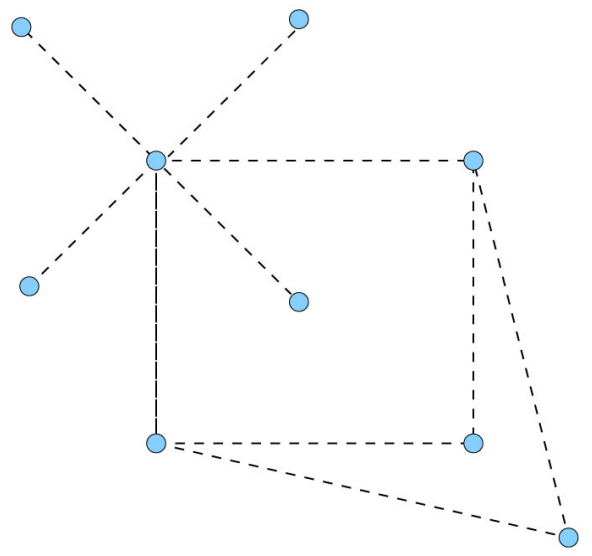

Figure 3. The graph $S_{4} \cdot C_{4} \cdot \cdot S_{2}$.

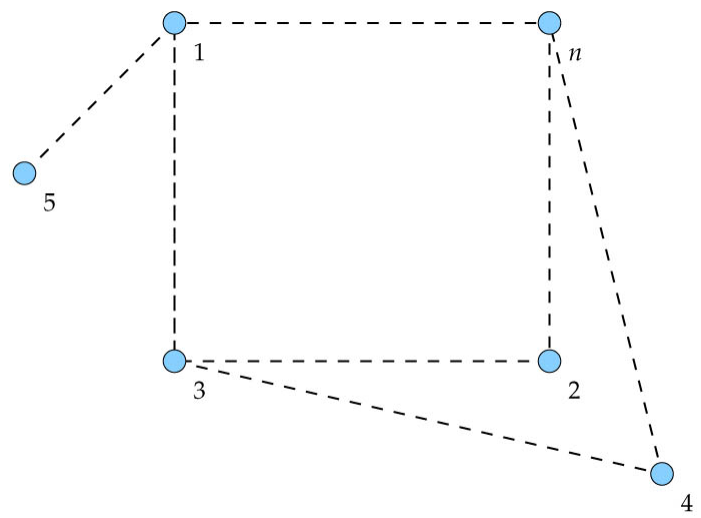

Figure 4. The graph $S_{2} \cdot C_{4} \cdot \cdot S_{2}$.

In each case $\beta$ can be extended in $(n-6)$ ! ways, giving the possible relative positions of the $(n-6) \quad x_{i}$ (see Figure 5). So, there are $4 \cdot 2 !(n-6)$ ! $\mathrm{RH}$ - configurations $(h, \beta)$.

The over graphs of $K_{n} \backslash\left(S_{1} \cdot C_{4} \cdot \cdot S_{2}\right)$ whose Ree-Hoover weight is not zero are up to isomorphism of the form: $K_{n} \backslash C_{4}, K_{n} \backslash\left(C_{4} \cdot S_{1}\right), K_{n} \backslash\left(C_{4} \cdot \cdot S_{2}\right)$, $K_{n} \backslash\left(S_{1} \cdot C_{4} \cdot \cdot S_{2}\right), \quad K_{n} \backslash\left(S_{1} \cdot C_{4} \cdot S_{1}\right), \quad K_{n} \backslash S_{l}, 1 \leq l \leq 3, K_{n} \backslash\left(S_{m}-S_{l}\right)$, $1 \leq m \leq 2,1 \leq l \leq 2$, and $K_{n}$. Their multiplicities are given by

$$
\begin{aligned}
\mid & w_{M}\left(K_{n} \backslash\left(S_{1} \cdot C_{4} \cdot \cdot S_{2}\right)\right) \mid \\
= & \left|w_{R H}\left(K_{n}\right)\right|+7\left|w_{R H}\left(K_{n} \backslash S_{1}\right)\right|+11\left|w_{R H}\left(K_{n} \backslash S_{2}\right)\right| \\
& +3\left|w_{R H}\left(K_{n} \backslash S_{3}\right)\right|+3\left|w_{R H}\left(K_{n} \backslash C_{4}\right)\right|+2\left|w_{R H}\left(K_{n} \backslash\left(S_{2}-S_{2}\right)\right)\right| \\
& +16\left|w_{R H}\left(K_{n} \backslash\left(S_{1}-S_{1}\right)\right)\right|+12\left|w_{R H}\left(K_{n} \backslash\left(S_{1}-S_{2}\right)\right)\right| \\
& +8\left|w_{R H}\left(K_{n} \backslash\left(C_{4} \cdot S_{1}\right)\right)\right|+\left|w_{R H}\left(K_{n} \backslash\left(C_{4} \cdot \cdot S_{2}\right)\right)\right| \\
& +\left|w_{R H}\left(K_{n} \backslash\left(S_{1} \cdot C_{4} \cdot \cdot S_{2}\right)\right)\right|+4\left|w_{R H}\left(K_{n} \backslash\left(S_{1} \cdot C_{4} \cdot S_{1}\right)\right)\right| .
\end{aligned}
$$

We conclude using Propositions (1), (2) and Propositions (19)-(23) of [3]. In the general case we have:

Proposition 3. For $j \geq 1, n \geq j+6$, we have, with the usual convention $\left(\begin{array}{c}j+1 \\ \ell\end{array}\right)=0$ if $\ell>j+1$, 

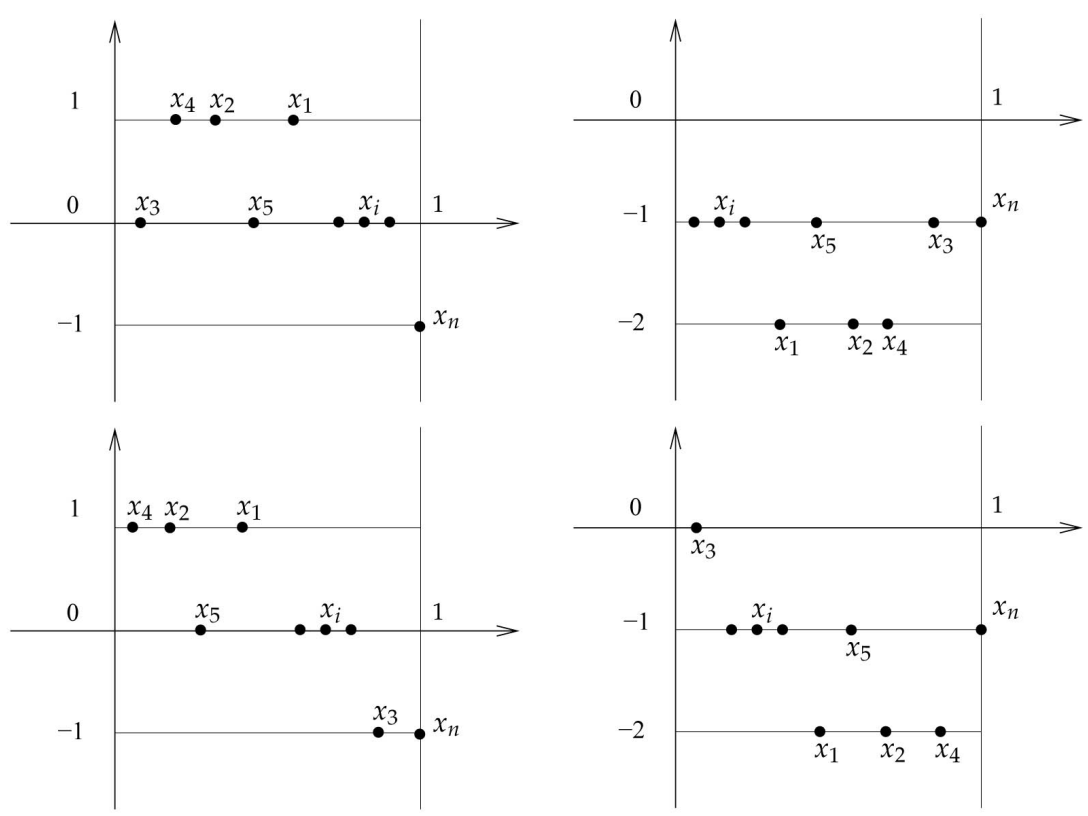

Figure 5. Fractional representation of a simplicial subpolytope of $\mathcal{P}_{R H}\left(K_{n} \backslash\left(S_{1} \cdot C_{4} \cdot S_{2}\right)\right)$.

$$
\begin{aligned}
& \left|w_{R H}\left(K_{n} \backslash\left(S_{j} \cdot C_{4} \cdot \cdot S_{2}\right)\right)\right|=\frac{8 j !}{(n-1)(n-2) \cdots(n-j-4)} . \\
\mid & w_{M}\left(K_{n} \backslash S_{j} \cdot C_{4} \cdot S_{2}\right) \mid \\
= & n+\frac{8}{n-1}+\frac{72}{(n-1)(n-2)(n-3)(n-4)} \\
& +\frac{32}{(n-1)(n-2)}+\frac{72}{(n-1)(n-2)(n-3)} \\
& +\sum_{l=1}^{j+2}\left[\left(\begin{array}{c}
j+2 \\
l
\end{array}\right) \frac{2 l !}{(n-1) \cdots(n-l)}+\left(\begin{array}{l}
j \\
l
\end{array}\right) \frac{8 l !}{(n-1) \cdots(n-l-2)}\right] \\
& +\sum_{l=1}^{j+2} 16\left(\begin{array}{l}
j \\
l
\end{array}\right)\left[\frac{2 l !}{(n-1) \cdots(n-l-3)}+\frac{l !}{(n-1) \cdots(n-l-4)}\right] \\
& +\sum_{l=1}^{j+2} 8\left(\begin{array}{c}
j \\
l-1
\end{array}\right)\left[\frac{l !}{(n-1) \cdots(n-l-2)}+\frac{l !}{(n-1) \cdots(n-l-3)}\right] .
\end{aligned}
$$

Proof. We can assume that the missing edges are $\{1, n\},\{2, n\},\{4, n\}$, $\{3,4\},\{2,3\},\{1,3\}$ and $\{1,5\},\{1,6\}, \ldots,\{1, j+4\}$ (see Figure 6, for the case of $\left.S_{2} \cdot C_{4} \cdot S_{2}\right)$.

According to Lemma 1 there are four possibilities for $h$ :

- $h_{1}=h_{2}=h_{4}=1$ and $h_{n}=-1$ and all other $h_{i}=0$, so that $\beta(3)=1$ and $(\beta(2), \beta(4))$ must be a permutation of $\{2,3\}$ and $(\beta(5), \beta(6), \ldots, \beta(j+4))$ must be a permutation of $\{4,5, \ldots, j+3\}$ and $\beta(1)=j+4$.

- $h_{1}=h_{2}=h_{4}=-2$ and all other $h_{i}=-1$, so that $\beta(3)=n-1$ and $(\beta(2), \beta(4))$ must be a permutation of $\{n-2, n-3\}$ and $(\beta(5), \beta(6), \ldots, \beta(j+4))$ must be a permutation of $\{n-4, n-5, \ldots, n-j-3\}$ and $\beta(1)=n-j-4$. 
- $h_{1}=h_{2}=h_{4}=1$ and $h_{3}=h_{n}=-1$ and all other $h_{i}=0$, so that $\beta(3)=n-1$ and $(\beta(2), \beta(4))$ must be a permutation of $\{1,2\}$ and $(\beta(5), \beta(6), \ldots, \beta(j+4))$ must be a permutation of $\{3,4, \ldots, j+2\}$ and $\beta(1)=j+3$.

- $h_{3}=0$ and $h_{1}=h_{2}=h_{4}=-2$ and all other $h_{i}=-1$, so that $\beta(3)=1$ and $(\beta(2), \beta(4))$ must be a permutation of $\{n-1, n-2\}$ and $(\beta(5), \beta(6), \ldots, \beta(j+4))$ must be a permutation of $\{n-3, n-4, \ldots, n-j-2\}$ and $\beta(1)=n-j-3$.

In each case $\beta$ can be extended in $(n-j-5))$ ! ways, giving the possible relative positions of the $(n-j-5) \quad x_{i}$ (see Figure 7, for the case of $\left.S_{2} \cdot C_{4} \cdot S_{2}\right)$. So, there are $4 \cdot 2 ! j !(n-j-5)$ ! RH-configurations $(h, \beta)$. Which concludes the proof of (2.8).

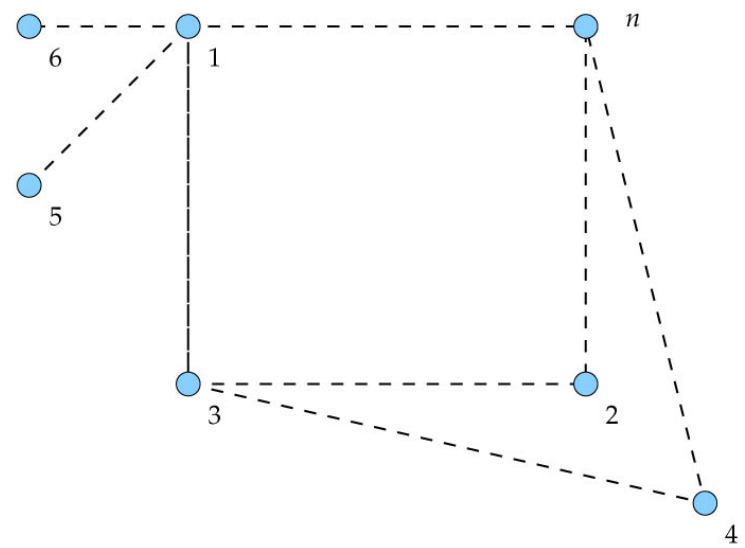

Figure 6. The graph $S_{2} \cdot C_{4} \cdot \cdot S_{2}$.
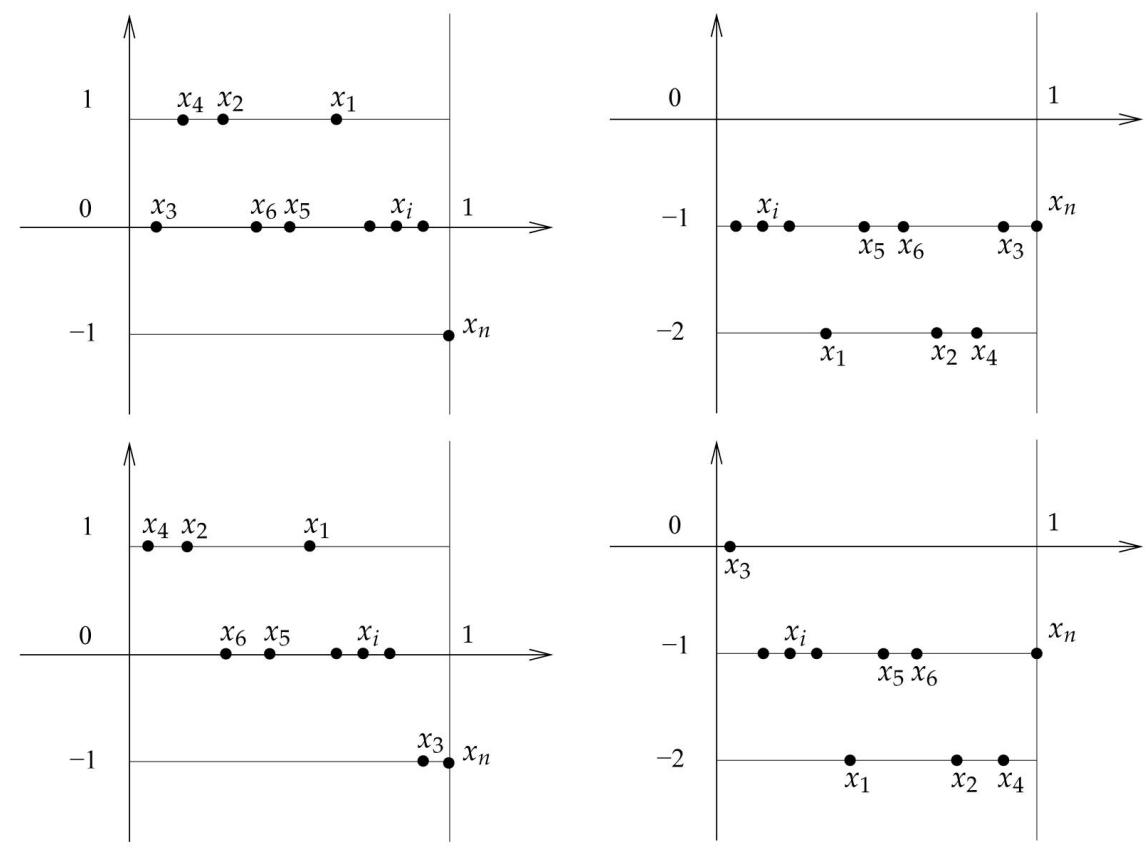

Figure 7. Fractional representation of a simplicial subpolytope of $\mathcal{P}_{R H}\left(K_{n} \backslash\left(S_{2} \cdot C_{4} \cdot \cdot S_{2}\right)\right)$. 
The over graphs of $K_{n} \backslash\left(S_{j} \cdot C_{4} \cdot S_{2}\right)$ whose Ree-Hoover weight is not zero and their multiplicities are given by

$$
\begin{aligned}
\mid & w_{M}\left(K_{n} \backslash\left(S_{j} \cdot C_{4} \cdot \cdot S_{2}\right)\right) \mid \\
= & \left|w_{R H}\left(K_{n}\right)\right|+4\left|w_{R H}\left(K_{n} \backslash S_{1}\right)\right|+8\left|w_{R H}\left(K_{n} \backslash S_{2}\right)\right|+2\left|w_{R H}\left(K_{n} \backslash S_{3}\right)\right| \\
& +3\left|w_{R H}\left(K_{n} \backslash C_{4}\right)\right|+12\left|w_{R H}\left(K_{n} \backslash S_{1}-S_{1}\right)\right|+6\left|w_{R H}\left(K_{n} \backslash S_{1}-S_{2}\right)\right| \\
& +\left|w_{R H}\left(K_{n} \backslash\left(C_{4} \cdot \cdot S_{2}\right)\right)\right|+6\left|w_{M}\left(K_{n} \backslash\left(C_{4} \cdot S_{1}\right)\right)\right| \\
& +\sum_{l=1}^{j+2}\left[\left(\begin{array}{c}
j+2 \\
l
\end{array}\right)\left|w_{R H}\left(K_{n} \backslash S_{l}\right)\right|+\left(\begin{array}{c}
j \\
l
\end{array}\right)\left|w_{R H}\left(K_{n} \backslash S_{l} \cdot C_{4} \cdot \cdot S_{2}\right)\right|\right] \\
& +\sum_{l=1}^{j} 2\left(\begin{array}{c}
j \\
l
\end{array}\right)\left[\left|w_{R H}\left(K_{n} \backslash C_{4} \cdot S_{l}\right)\right|+2\left|w_{R H}\left(K_{n} \backslash S_{1} \cdot C_{4} \cdot S_{l}\right)\right|\right] \\
& +\sum_{l=1}^{j+2} 2\left[\left(\begin{array}{l}
j \\
l
\end{array}\right)+\left(\begin{array}{c}
j \\
l-1
\end{array}\right)\right]\left[\left|w_{R H}\left(K_{n} \backslash S_{2}-S_{l}\right)\right|+2\left|w_{R H}\left(K_{n} \backslash S_{1}-S_{l}\right)\right|\right] .
\end{aligned}
$$

We conclude using Propositions (1), (3) and Propositions (19)-(23) of [3].

\section{Conflicts of Interest}

The author declares no conflicts of interest regarding the publication of this paper.

\section{References}

[1] Kaouche, A. (2010) Invariants de graphes liés aux gaz imparfaits. Publications du Laboratoire de Combinatoire et d Informatique Mathematique (LaCIM), 42.

[2] Kaouche, A. and Leroux, P. (2008) Graph Weights Arising from Mayer and Ree-Hoover Theories. DMTCS Proceedings, 20 th Annual International Conference on Formal Power Series and Algebraic Combinatorics (FPSAC 2008), 259-270.

[3] Kaouche, A. and Leroux, P. (2009) Mayer and Ree-Hoover Weights of Infinite Families of 2-Connected Graphs. Séminaire Lotharingien de Combinatoire 61 A, Article B61Af.

[4] Labelle, G., Leroux, P. and Ducharme, M.G. (2007) Graph Weights Arising from Mayer's Theory of Cluster Integrals. Séminaire Lotharingien de Combinatoire, 54, Article B54m.

[5] Uhlenbeck, G.E. and Ford, G.W. (1963) Lectures in Statistical Mechanics. American Mathematical Society, Providence, $181 \mathrm{p}$.

[6] Ree, F.H. and Hoover, W.G. (1964) Fifth and Sixth Virial Coefficients for Hard Spheres and Hard Discs. The Journal of Chemical Physics, 40, 939-950. https://doi.org/10.1063/1.1725286

[7] Ree, F.H. and Hoover, W.G. (1964) Reformulation of the Virial Series for Classical Fluids. The Journal of Chemical Physics, 41, 1635-1645. https://doi.org/10.1063/1.1726136

[8] Ree, F.H. and Hoover, W.G. (1967) Seventh Virial Coefficients for Hard Spheres and Hard Discs. The Journal of Chemical Physics, 46, 4181-4196. https://doi.org/10.1063/1.1840521

[9] Clisby, N. and McCoy, B.M. (2004) Negative Virial Coefficients and the Dominance of Loose Packed Diagrams for D-Dimensional Hard Spheres. Journal of Statistical Physics, 114. https://doi.org/10.1023/B:JOSS.0000013960.83555.7d

[10] Clisby, N. and McCoy, B.M. (2006) Ninth and Tenth Order Virial Coefficients for 
Hard Spheres in D Dimensions. Journal of Statistical Physics, 122, 15-57. https://doi.org/10.1007/s10955-005-8080-0

[11] Kaouche, A. (2016) Valeurs des poids de Mayer et des poids de Ree-Hoover pour tous les graphes 2-connexes de taille au plus 8 et leurs parametres descriptifs.

http://professeure.umoncton.ca/umce-kaouche_amel/files/umce-kaouche_amel/wf/ wf/TableauRH7.pdf

http://professeure.umoncton.ca/umce-kaouche_amel/files/umce-kaouche_amel/wf/ wf/TableauRH8.pdf

[12] Kaouche, A. and Labelle, G. (2008) Mayer Polytopes and divided differences, Congrès Combinatorial Identities and Their Applications in Statistical Mechanics, Isaac Newton Institute de l'Université de Cambridge, en Angleterre.

[13] Kaouche, A. and Labelle, G. (2013) Mayer and Ree-Hoover Weights, Graphs Invariants and Bipartite Complete Graphs. Pure Mathematics and Applications, 24, 19-29.

[14] Kaouche, A., Labelle, G. (2014) Poids de Mayer et transformées de Fourier. Annales Mathématiques du Québec Springer-Verlag, 38, 37-59.

https://doi.org/10.1007/s40316-014-0018-y 